Session 2: The natal precursors of OB stars 


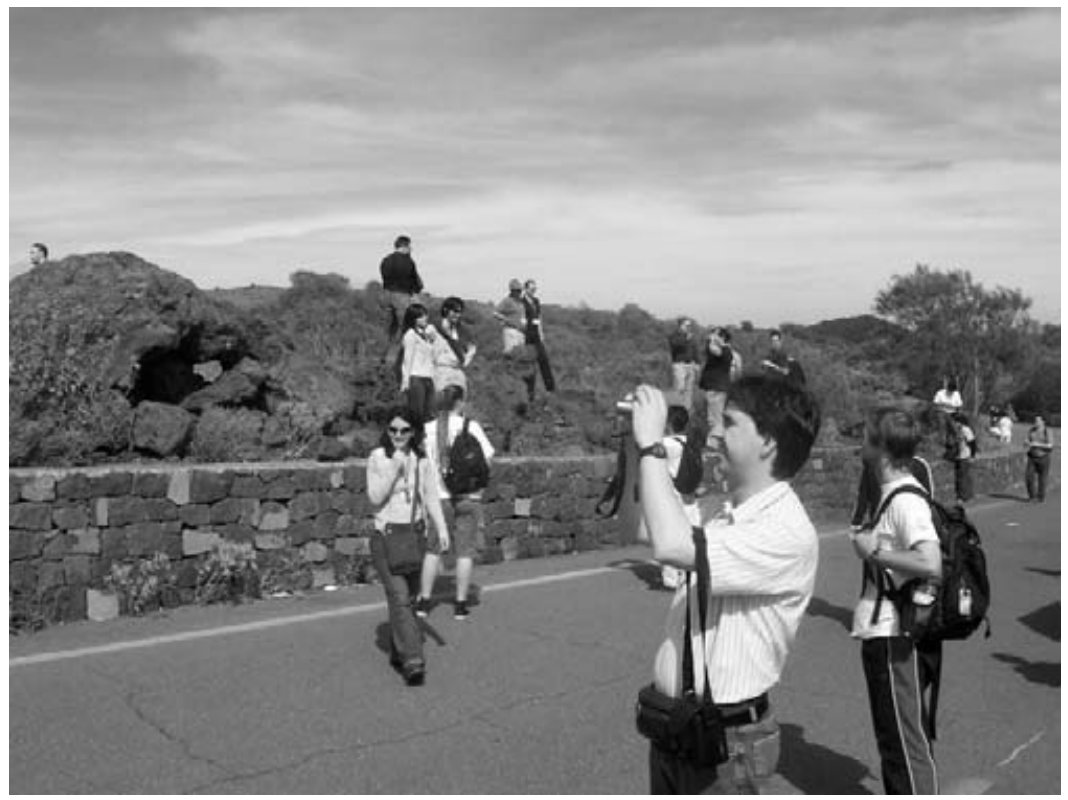

Excursion to Etna

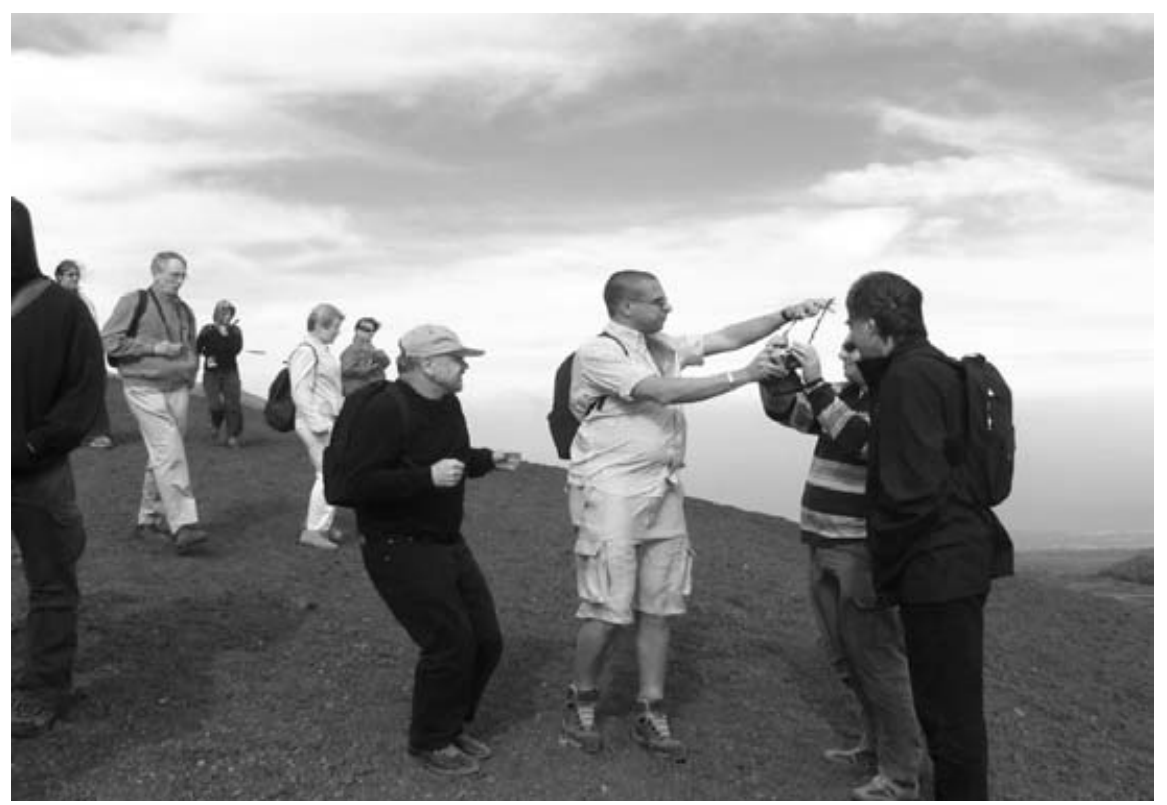

Excursion to Etna 


\title{
Ices as tracers of massive star birth
}

\author{
M. Elisabetta Palumbo \\ INAF Osservatorio Astrofisico di Catania, Via Santa Sofia 78, I-95123 Catania, Italy \\ email: mepalumbo@ct.astro.it
}

\begin{abstract}
The presence of ices along the line of sight of high mass star forming regions is clearly evidenced by infrared observations. In dense molecular clouds icy grain mantles form after direct freeze out of gas phase species and after surface reactions of atoms and radicals on grains. Thus chemical composition of icy mantles differs from that of the gas phase. Due to the presence of the protostar and of cosmic radiation, icy mantles suffer from ion bombardment, UV photolysis and thermal annealing. Most of our knowledge on the physical and chemical properties of ices is based on the comparison between observations and laboratory experiments performed at low temperature $(10-80 \mathrm{~K})$. Experimental results show that after ion irradiation and UV photolysis the chemical composition and the structure of the sample is modified. Both more volatile and less volatile species are formed and if a C-bearing species is present in the original sample a refractory residue is left over after warm-up to room temperature. After thermal annealing, segregation, crystallization, and sublimation take place. Thus molecular species are released to the gas phase which could be enriched by species formed in the solid phase. Here I will discuss some recent laboratory experiments relevant to our knowledge of the physico-chemical properties of ices in star forming regions.
\end{abstract}

Keywords. Astrochemistry, molecular processes, methods: laboratory, techniques: spectroscopic, stars: formation, ISM: molecules, infrared: ISM.

\section{Introduction}

In dense molecular clouds $\left(n>10^{4} \mathrm{~cm}^{-3}\right)$ gas phase species condense on silicatic and carbonaceous grains giving rise to icy mantles. These mantles are made of molecules which directly freeze out from the gas phase (such as $\mathrm{CO}$ ) and molecules which are formed after grain surface reactions (such as $\mathrm{H}_{2} \mathrm{O}$ ). The presence of icy grain mantles is indirectly deduced from depletion of gas phase species and observed in the infrared from absorption features attributed to vibrational modes of solid phase molecules superposed to the background stellar spectrum. Ices have been observed in star forming regions (both low- and high-mass) as well as in quiescent dense clouds.

Solid objects in space (interstellar grains, comets, interplanetary dust particles), are continuously exposed to energetic processes such as cosmic ray irradiation and UV photolysis. Fast ions passing through a molecular solid release energy to the target material. As a consequence many molecular bonds are broken along the ion-track and, in a very short time (one picosec or less), the molecular fragments recombine giving rise to a rearrangement of the chemical structure. In addition to the alteration of the chemical and lattice structure of the target material, new molecular species (not present before irradiation) are formed. In the case of UV photolysis, the energy is released to the target material through a single photo-dissociation or photo-excitation event. Also in this case new molecular species are formed.

Following the approximation of monoenergetic $1 \mathrm{MeV}$ protons it has been estimated that the proton flux values 1.8 protons $\mathrm{cm}^{-2} \mathrm{~S}^{-1}$ in diffuse quiescent regions and 1 proton $\mathrm{cm}^{-2} \mathrm{~s}^{-1}$ in dense quiescent regions (Mennella et al. 2003). Estimates of the UV flux 
under diffuse medium conditions give a value of $8 \times 10^{7}$ photons $\mathrm{cm}^{-2} \mathrm{~s}^{-1}$ (Mathis et al. 1983). In dense regions the internal UV flux due to cosmic-ray induced fluorescence of molecular hydrogen values $1.4 \div 4.8 \times 10^{3}$ photons $\mathrm{cm}^{-2} \mathrm{~s}^{-1}$ (Prasad \& Tarafdar 1983; Mennella et al. 2003). Taking into account the amount of energy released by ions and photons passing through an ice mantle, the effects of UV photons dominate in diffuse regions while the effects of ions and UV photons are comparable in dense regions (Moore 1999). As reported by Greenberg (1982), dense cloud lifetime has been estimated at $3 \times 10^{7}-5 \times 10^{8}$ years. Assuming $n \sim 10^{4} \mathrm{~cm}^{-3}$, the gas takes $10^{9} / n \simeq 10^{5}$ years to condense on grains (Tielens \& Allamandola 1987). Thus icy grain mantles suffer ion irradiation for about $10^{5}-5 \times 10^{8}$ years . The former estimate refers to the case of grain mantles which sublimate immediately after formation while the latter refers to the limit case of icy grain mantles (or at least part of them) which survive for all the cloud lifetime. The specific energy loss (stopping power) of $1 \mathrm{MeV}$ protons in a typical grain containing heavy atoms $(\mathrm{C}, \mathrm{N}, \mathrm{O}, \mathrm{Si})$ is $S \simeq 5 \times 10^{-15} \mathrm{eV} \mathrm{cm}^{2}$ atom ${ }^{-1}$. Thus the energy deposited on a grain (dose), given by the product of the stopping power times the flux times the mantle lifetimes $\left(3 \times 10^{12}-1.5 \times 10^{16} \mathrm{~s}\right)$, values $0.015-75 \mathrm{eV} /$ atom $(\mathrm{C}, \mathrm{N}, \mathrm{O}, \mathrm{Si})$. Of course in star forming regions the flux of ions and then the doses absorbed by icy mantles can be very different from the values reported above.

\section{Experimental methods}

Several laboratories worldwide are involved in the study of astrophysical relevant ices and of the modifications induced by ion irradiation, UV photolysis and thermal annealing. Here I will describe in detail the experimental set-up available at the Laboratory of Experimental Astrophysics (L.A.SP.) in Catania (Italy). A schematic view of the experimental apparatus is reported in Fig. 1.

In situ IR spectroscopy is performed in a stainless steel vacuum chamber facing an FTIR spectrometer (Bruker Equinox 55). Inside the vacuum chamber, in which pressure is kept below $10^{-7}$ mbar, an IR transparent substrate (e.g. crystalline silicon) is placed in thermal contact with a cold finger which temperature can be varied between $10 \mathrm{~K}$ and $300 \mathrm{~K}$. The vacuum chamber is interfaced with an ion implanter (200 kV; Danfysik) from which ions with energy up to $200 \mathrm{keV}$ (400 keV for double ionizations) can be obtained. The ion beam produce a $2 \times 2 \mathrm{~cm}^{2}$ spot on the targets and current in the range of $100 \mathrm{nA}$ $\mathrm{cm}^{-2}$ to a few $\mu \mathrm{A} \mathrm{cm}^{-2}$.

A hydrogen microwave discharge resonance lamp (Opthos Instruments) is interfaced with the vacuum chamber through an $\mathrm{MgF}_{2}$ window; from this lamp mainly $10.2 \mathrm{eV}$ photons are obtained. An aluminium light collector is placed at the end of the lamp in order to increase the number of UV photons that reaches the sample. A light detector, placed at the end of the aluminium light collector, is used to measure the UV flux during photolysis. The detector is a platinum wire which gives a current, by the photoelectric effect, proportional to the UV flux. The wire detector has been calibrated using the procedure described in details by Westley et al. (1995a,b) and Baratta et al. (2002). It is important to consider that the discharge lamps are not monochromatic, with a spectrum of the emitted radiation depending on experimental conditions, such as pressure in the discharge tube and gas mixture ratio. For example, the operating conditions adopted by Westley et al. (1995b; see their Fig. 1) produce negligible UV radiation outside the Lyman- $\alpha$ line; on the other hand, the Lyman- $\alpha$ emission accounts for at most $5 \%$ of the total lamp intensity in the operating conditions used by Cottin et al. (2003; see their Fig. 1). The operating conditions of the UV lamp adopted in our laboratory are very similar to those used by Westley et al. $(1995 \mathrm{a}, \mathrm{b})$, and thus we assume that we have a 


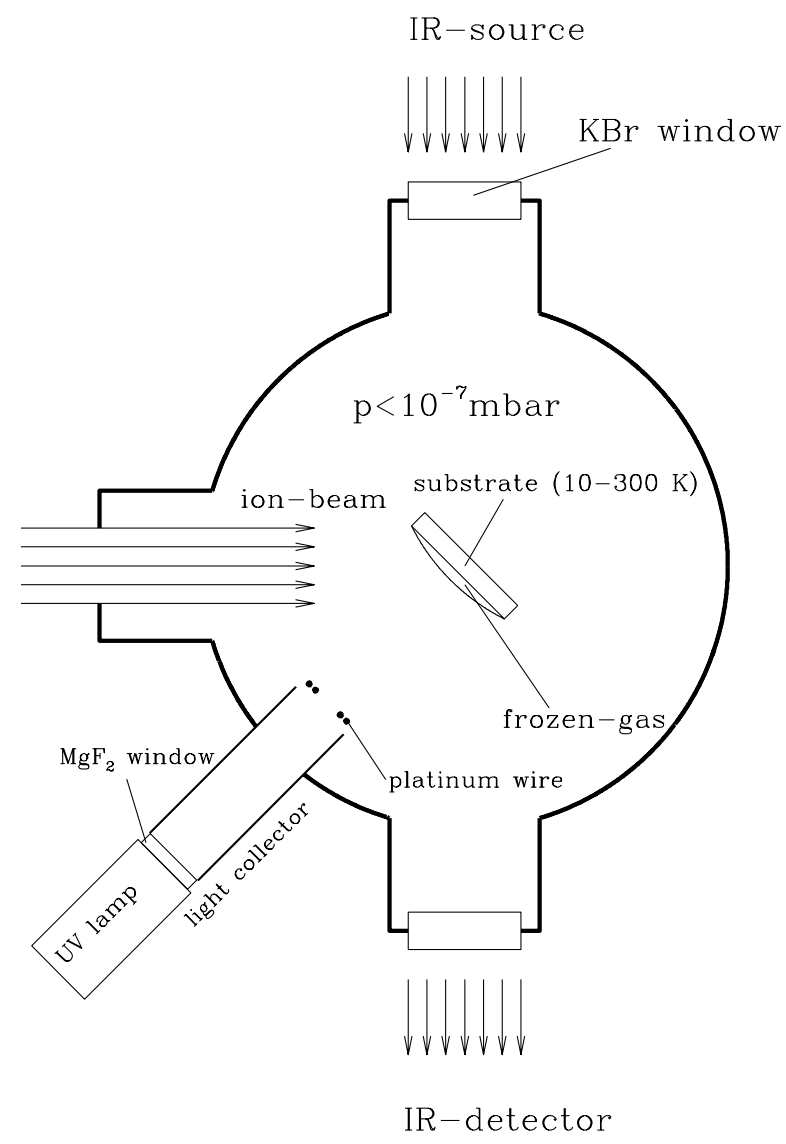

Figure 1. Schematic view of the experimental apparatus used for in situ IR spectroscopy of ion irradiated and UV photolyzed frozen gases and solids.

similar spectrum. However we cannot exclude the possibility that lower energy photons are present. Cottin et al. (2003) have estimated that in their experimental conditions the average photon energy is about $7.4 \mathrm{eV}$. At any rate, this would introduce an uncertainty of at most $30 \%$ in the energy dose calculations.

The amount of energy released to the sample (dose) is often expressed in eV per small molecule (16 a.m.u.) because this is a convenient way to characterize the chemical changes and to compare the effects induced on icy mixtures with different chemical composition (Strazzulla \& Johnson 1991). In the case of ion irradiation the dose is calculated from the knowledge of the ion fluence (ions $\left.\mathrm{cm}^{-2}\right)$, the stopping power $\left(\mathrm{eV} \mathrm{cm}^{2}\right.$ molecules $\left.{ }^{-1}\right)$ of the chosen projectile, and its penetration depth or range in the target $\left(\right.$ molecules $\left.\mathrm{cm}^{-2}\right)$. The first is given by a current integrator on the path of the ion beam, which measures the charge which reaches the sample during irradiation; the other two parameters are well known and can be provided by a software such as the TRIM Montecarlo simulation program (Ziegler et al. 1985). In the case of UV photolysis the dose can be calculated from the knowledge of the UV fluence (photons $\mathrm{cm}^{-2}$ ) and the absorption coefficient of 
UV photons in ices which can be measured experimentally as described by Baratta et al. (2002).

A needle valve is used to admit pre-prepared gas (or mixtures) into the chamber, where they freeze on the substrate. A He-Ne laser can be used to monitor the thickness of the ice film during accretion; this is achieved by looking at the interference pattern (intensity versus time) given by the laser beam reflected at an angle of $45^{\circ}$ both by the vacuum-film and film-substrate interfaces (see Baratta \& Palumbo (1998) for further details on the technique used to measure the thickness).

The substrate holder is mounted at an angle of $45^{\circ}$ with respect to both the ion beam and the IR beam and it is orthogonal to the UV beam, so that spectra can be easily taken in situ, even during irradiation with ions and UV photons, without tilting the sample. For this purpose the IR spectrometer is positioned (by a moveable optical bench) such that the IR beam is transmitted, through a hole in the sample holder, by the substrate. In addition to unpolarized transmission spectra at an incidence angle of $45^{\circ}$, spectra with the electric vector parallel ( $\mathrm{P}$ polarized) and perpendicular ( $\mathrm{S}$ polarized) to the plane of incidence can be taken. The plane of incidence is the plane of the paper in Fig. 1; this plane contains $\mathrm{P}$ polarized light while the plane of $\mathrm{S}$ polarization is perpendicular to the paper. The polarization of the incident radiation can be changed rotating a polarizer (not shown) placed in the path of the infrared beam (Baratta \& Palumbo 1998). As discussed by Baratta et al. (2000) spectra taken at oblique incidence in S polarization are equivalent to unpolarized spectra at normal incidence.

\section{Results and discussion}

The effects induced by fast ions and UV photons on solids of astrophysical interest have been separately studied in different laboratories for many years (e.g., Hagen et al. 1979; Brown et al. 1982; Moore et al. 1983; Strazzulla et al. 1983; Allamandola et al. 1988; Baratta et al. 1991; Westley et al. 1995a,b; Gerakines et al. 1996; Strazzulla et al. 2001; Moore et al. 2001). It is well known that both processes induce chemical (e.g., Moore et al. 1983; Gerakines et al. 1996) and structural (e.g., Baratta et al. 1991; Moore \& Hudson 1992; Leto \& Baratta 2003) modifications of the ice samples. Only recently a few laboratories have been equipped in order to study the effects induced in frozen gases and solids by both fast ions and UV photons (Gerakines et al. 2000; Cottin et al. 2001; Gerakines \& Moore 2001; Baratta et al. 2002; Hudson \& Moore 2002; Leto \& Baratta 2003; Gerakines et al. 2004). This can be done: (a) comparing the results of separate experiments; (b) switching from one energy source to the other during the same experiment; (c) irradiating the sample simultaneously with ions and UV photons. Figures 2 and 3 show the effects of UV photolysis and ion irradiation on a sample of pure methanol ice at low temperature $(10-12 \mathrm{~K})$. The effects of ion irradiation and UV photolysis on pure solid methanol have been separately studied in different laboratories (Gerakines et al. 1996; Palumbo et al. 1999; Hudson \& Moore 2000). Recently these effects have been studied in the same experimental set-up by Baratta et al. (2002) and a quantitative comparison has been performed. It has been shown that in both cases the column density of methanol decreases while the column density of newly formed species such as carbon monoxide $(\mathrm{CO})$, carbon dioxide $\left(\mathrm{CO}_{2}\right)$, methane $\left(\mathrm{CH}_{4}\right)$ and formaldehyde $\left(\mathrm{H}_{2} \mathrm{CO}\right)$ increases during irradiation and as irradiation proceeds further the column density of these species decreases too. It is interesting to note that at the lowest doses the effects induced by ions and UV photons are quantitatively very similar while at higher doses some differences occur. In fact both processes modify the optical properties of the icy samples. However, while ions release energy to the sample independently of its optical properties, 


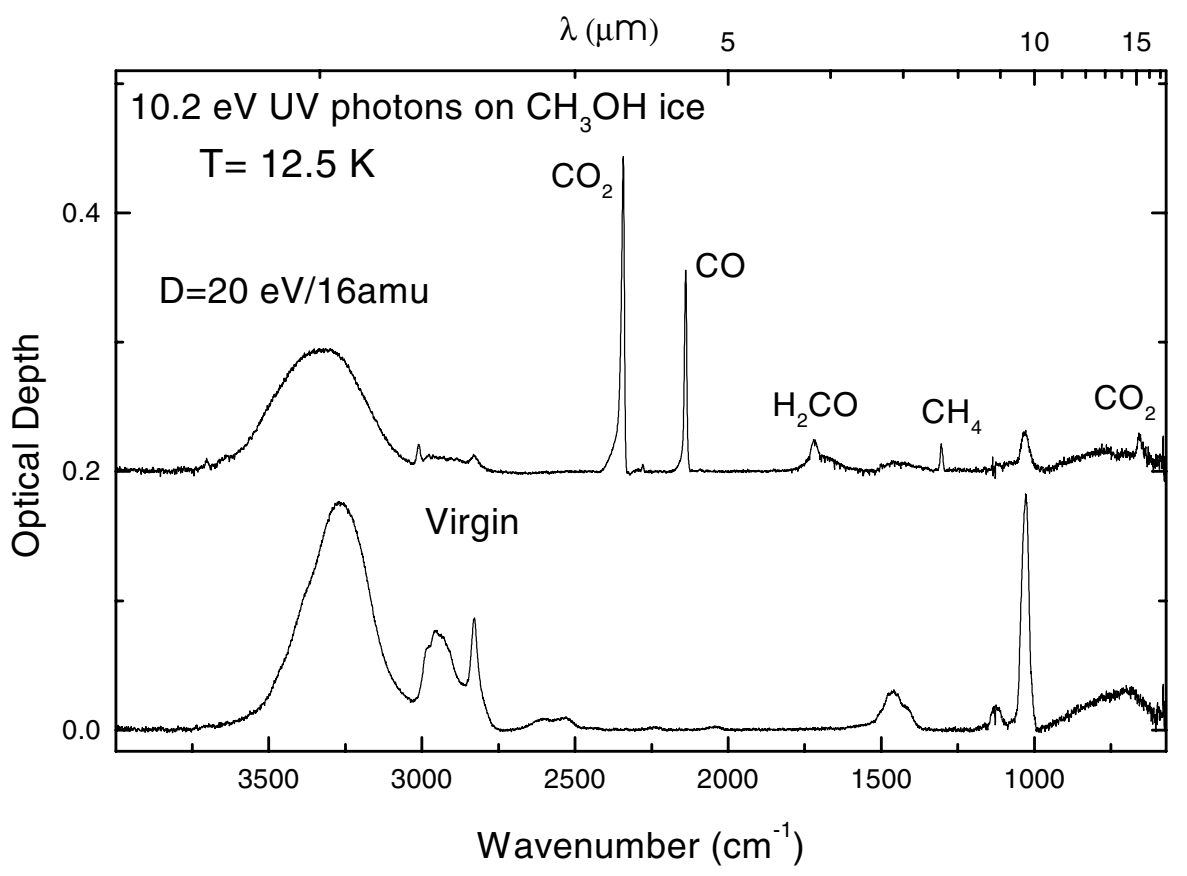

Figure 2. Infrared spectra of pure methanol deposited on a cold (12.5 K) substrate before and after irradiation with $10.2 \mathrm{eV}$ Lyman- $\alpha$ photons (dose of $20 \mathrm{eV} / 16 \mathrm{amu}$ ). The spectra have been arbitrarly shifted in optical depth scale for clarity.

the interaction of UV photons with matter strongly depends on its optical properties. In the case of methanol the effects of ion irradiation and UV photolysis are comparable on the fresh ice (i.e. at the lowest doses) while they are very different as soon as the original ice sample is modified by the processing itself (i.e. at the highest doses). This difference is clearly evident in hydrocarbon-rich icy samples (Baratta et al. 2002) while it has not been observed after comparison of the effects of ion irradiation and UV photolysis on pure CO ice (Loeffler et al. 2005).

Several effects are induced by thermal annealing of icy samples. It has been shown that when an ice mixture made of $\mathrm{H}_{2} \mathrm{O}, \mathrm{CH}_{3} \mathrm{OH}$ and $\mathrm{CO}_{2}$ is warmed-up, a modification of the profile of the $\mathrm{CO}_{2}$ bands is observed which is interpreted as segregation (e.g., Ehrenfreund et al. 1998; Palumbo \& Baratta 2000). Furthermore crystallization of amorphous samples is induced after thermal annealing. In the case of water, laboratory experiments have shown that different forms of ice exist, as a consequence of deposition rate of the vapor, substrate temperature, thermal and irradiation history. Crystalline hexagonal ice is formed at temperature higher than $150 \mathrm{~K}$ and crystalline cubic ice is formed between 110 and $150 \mathrm{~K}$. When vapor is deposited at temperatures lower than $100 \mathrm{~K}$, the resulting ice is amorphous and different forms of amorphous water ice have been reported. Amorphous ice is converted to crystalline ice as the temperature increases while crystalline ice is stable when temperature decreases. Crystalline ice is converted to amorphous ice after ion irradiation and UV photolysis (e.g., Baratta et al. 1991; Moore \& Hudson 1992; Leto \& Baratta 2003). 
Eventually, after thermal annealing, sublimation of icy samples is observed. It has been shown that sublimation temperature of each single species also depends on the ice mixture it is embedded in (e.g., Bar-Nun et al. 1985; Collings et al. 2004). Furthermore the sublimation process can be modified if the ice is irradiated at low temperature. In particular, Fig. 3 shows a comparison between infrared spectra of pure methanol taken at different temperatures $(\mathrm{T}=10,100,150,200 \mathrm{~K})$ with those of methanol irradiated at $10 \mathrm{~K}$ with $3 \mathrm{keV} \mathrm{He}{ }^{+}$ions (dose of $52 \mathrm{eV} / 16 \mathrm{amu}$ ) and warmed up. It is evident that pure methanol sublimes between 150 and $200 \mathrm{~K}$, while after ion irradiation methanol is still partially present at $200 \mathrm{~K}$. Similarly, Fig. 4 shows the sublimation rate, at $35 \mathrm{~K}$, of pure CO ice and of CO after ion irradiation with $3 \mathrm{keV} \mathrm{He}^{+}$ions (dose of $11 \mathrm{eV} / 16 \mathrm{amu}$ ). Again the sublimation rate is modified after ion irradiation. In particular it has been estimated, from the variation of the integrated intensity of the $2140 \mathrm{~cm}^{-1} \mathrm{CO}$ band, that the sublimation rate values $19.2 \AA \mathrm{s}^{-1}$ for pure CO ice and $2.1 \AA \mathrm{s}^{-1}$ after ion irradiation (Baratta et al. 1994). The different sublimation behaviour of ion irradiated ices with respect to pure ices is attributed to the formation, after irradiation, of less volatile species (residue) which trap the original volatiles. In fact formation of suboxides has been clearly evidenced after ion irradiation of pure CO ice (e.g., Strazzulla et al. 1997; Brucato et al. 1997; Gerakines \& Moore 2001). In the case of methanol a refractory residue stable at room temperature is formed (Ferini et al. 2004; Palumbo et al. 2004)

Recently an experimental study has compared the information obtained by infrared and Raman spectra of ion irradiated icy mixtures (e.g., Palumbo et al. 2004 and references therein). All the samples have been irradiated at low temperature (about $10 \mathrm{~K}$ ), then warmed up and irradiated again at room temperature. Infrared spectroscopy shows that several new absorption features appear after ion irradiation at low temperature, indicating the formation of new molecular species. After warm-up, volatile species sublimate and a refractory residue is left over at room temperature. After further irradiation of the residue at room temeprature, the intensity of all infrared bands decreases and a featureless spectrum is obtained. Raman spectroscopy of similar mixtures shows that the residue is already formed at low temperature and that ion irradiation causes a modification of the structure of the samples which evolve towards an amorphous carbon.

Both ground-based and airborne observations of dense molecular clouds clearly indicate the presence of ices along the line of sight of many embedded young stellar objects spanning a wide range of mass and luminosity. In particular, solid water $\left(\mathrm{H}_{2} \mathrm{O}\right)$, carbon monoxide $(\mathrm{CO})$, carbon dioxide $\left(\mathrm{CO}_{2}\right)$, methanol $\left(\mathrm{CH}_{3} \mathrm{OH}\right)$ and methane $\left(\mathrm{CH}_{4}\right)$ have been securely identified. Other species such as carbonyl sulphide (OCS), formaldeyde $\left(\mathrm{H}_{2} \mathrm{CO}\right)$, formic acid $(\mathrm{HCOOH})$, cyanate ion $\left(\mathrm{OCN}^{-}\right)$and ammonia $\left(\mathrm{NH}_{3}\right)$ have been likely identified. Some features, observed in the astronomical spectra, still remain unidentified (see Gibb et al. 2004 for a recent review). Along all the lines of sight water is always the most abundant solid phase species. The abundance of solid $\mathrm{CO}_{2}$ ranges between $10 \%$ to $25 \%$ with respect to solid water (Gerakines et al. 1999), the abundance of $\mathrm{CH}_{3} \mathrm{OH}$ values 3-30\% (Allamandola et al. 1992; Dartois et al. 1999a), the abundance of $\mathrm{CH}_{4}$ ranges between $0.3 \%$ and $4 \%$ (e.g., Boogert et al. 1997), solid CO can reach an abundance up to $40 \%$ with respect to water ice (e.g., Chiar et al. 1994).

In the last decades the comparison between laboratory experiments and astronomical observations had a fundamental role in our knowledge of the physico-chemical properties of icy mantles. As an example the profile of solid $\mathrm{CO}_{2}$ bands observed in dense molecular clouds has been compared with laboratory spectra. In particular the asymmetric profile of the bending mode at about $15 \mu \mathrm{m}\left(660 \mathrm{~cm}^{-1}\right)$ observed towards high mass star forming regions shows the presence of segregated $\mathrm{CO}_{2}$ which has been interpreted as evidence of thermal annealing along the line of sight (e.g., Ehrenfreund et al. 1998; Gerakines 

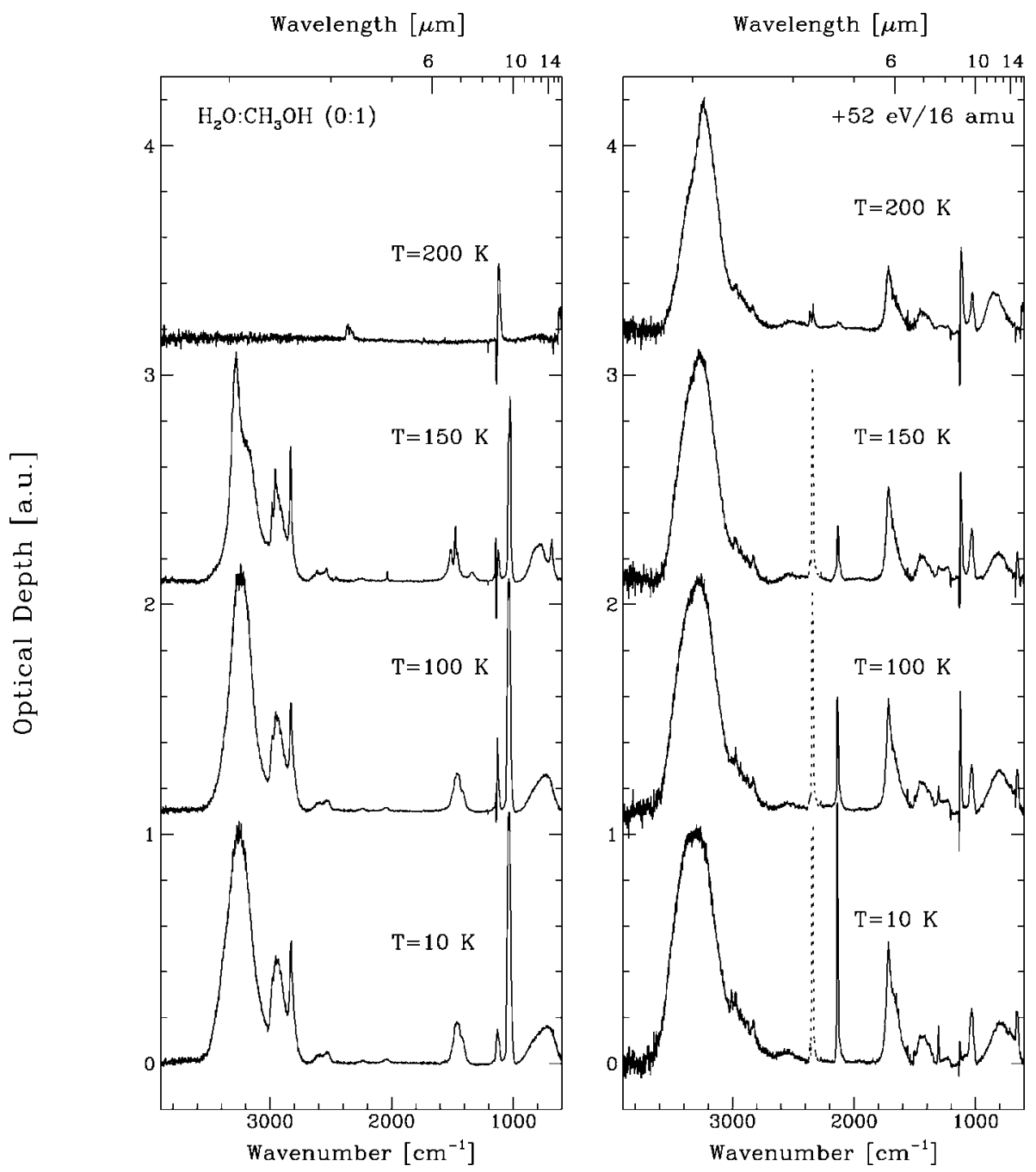

Figure 3. Infrared spectra of unirradiated pure methanol are compared, at different temperatures $(\mathrm{T}=10,100,150,200 \mathrm{~K})$ with those of methanol irradiated with $3 \mathrm{keV} \mathrm{He}^{+}$ions (dose of $52 \mathrm{eV} / 16 \mathrm{amu}$ ). All the spectra have been normalized to the $\mathrm{O}-\mathrm{H}$ streching band at about 3260 $\mathrm{cm}^{-1}$. The profile of the $\mathrm{CO}_{2}$ band at about $2340 \mathrm{~cm}^{-1}$, because of its high intensity (about twice that of the $3260 \mathrm{~cm}^{-1}$ band), has been plotted for clarity, up to optical depth equal to 1 (dotted lines).

et al. 1999). Furthermore from a detailed comparison between observed and laboratory spectra it has been evidenced the presence of $\mathrm{CO}_{2}-\mathrm{CH}_{3} \mathrm{OH}$ intermolecular complexes in icy mantles (Dartois et al. 1999b). Recent Spitzer spectra in the range of the $15 \mu \mathrm{m} \mathrm{CO}_{2}$ feature towards field stars do not show any evidence of segregation and then thermal annealing along these lines of sight (Bergin et al. 2005). Thus segregation is due to thermal processing induced by the forming star embedded in its parental cloud. Based on ISO observations of the $3.1 \mu \mathrm{m}\left(3300 \mathrm{~cm}^{-1}\right)$ water ice band along many protostellar lines of sight, it has been evidenced that in some instances the profile of this feature is very similar to the profile of amorphous solid water while in other cases it resembles laboratory spectra of crystalline water ice (Dartois \& d'Hendecourt 2001). It is generally accepted that water ice in dense molecular clouds forms because of surface reactions on 


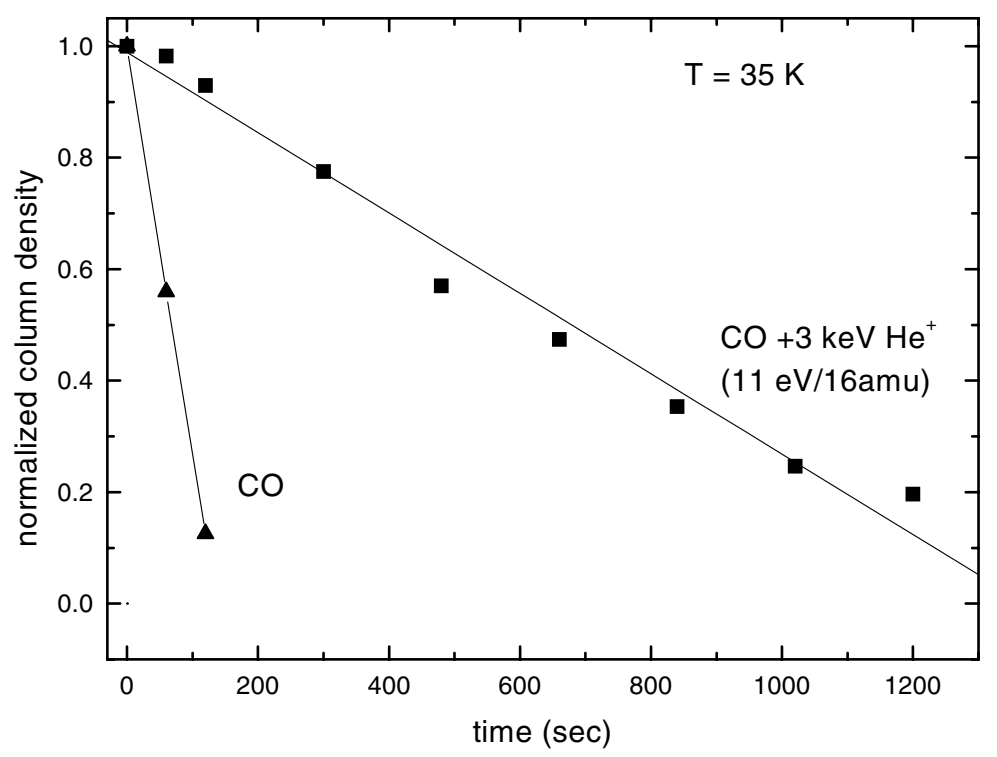

Figure 4. Sublimation rate of a $\mathrm{CO}$ ice film without processing (triangles) and after ion irradiation with $3 \mathrm{keV} \mathrm{He}^{+}$ions (dose $=11 \mathrm{eV} / 16 \mathrm{amu}$; squares).

grain mantles at low temperature (about $10 \mathrm{~K}$ ). Thus interstellar water ice is believed to be amorphous (Hagen et al. 1981). Then the evidence of crystalline water ice along many lines of sight can only be due to the effects of thermal annealing caused by the embedded young stellar object.

Several questions about ices in star forming regions remain unclear. Among these the origin of solid carbon dioxide. The low abundance of this molecule in the gas phase excludes the possibility that solid $\mathrm{CO}_{2}$ is the result of direct freeze out. Thus solid $\mathrm{CO}_{2}$ can be formed in grain mantles after ion irradiation and/or UV photolysis. Loeffler et al. (2005) have estimated that an amount between $1 \%$ to $6 \%$ of the observed solid carbon dioxide can be formed after processing of CO-rich icy mantles while an amount ten times higher can be formed in $\mathrm{H}_{2} \mathrm{O}$-rich icy mantles (i.e. $\mathrm{H}_{2} \mathrm{O}: \mathrm{CO}=10: 1$; Watanabe \& Kouchi 2002; Mennella et al. 2004). It has also been suggested that in dense clouds solid carbon dioxide can be formed after cosmic ray irradiation of carbon grains with a water ice cap (Mennella et al. 2004). The comparison between laboratory results and observations indicates that the amount of $\mathrm{CO}_{2}$ produced by cosmic-ray irradiation of carbon particles can account for 3\%-7\% of the observed column densities. Alternatively, observed solid carbon dioxide could be the result of grain surface reactions involving $\mathrm{CO}$ and atomic $\mathrm{O}$ (Roser et al. 2001).

Finally, it is worth mentioning that some molecules such as acetaldeyde $\left(\mathrm{CH}_{3} \mathrm{CHO}\right)$, ethylene oxide $\left(\mathrm{c}_{-} \mathrm{C}_{2} \mathrm{H}_{4} \mathrm{O}\right)$ and vinyl alcohol $\left(\mathrm{HCOOCH}_{3} ; \mathrm{Nummelin}\right.$ et al. 1998; Ikeda et al. 2001) and methyl formate (Horn et al. 2004) have been observed in the gas phase and their abundances cannot be explained only by gas phase chemistry (see van der Tak, this volume). It has been suggested that these molecules could be formed after energetic processing of icy grain mantles and be released to the gas phase after sublimation of icy mantles. Next generation of laboratory experiments will try to answer to these questions. 


\section{Acknowledgements}

Lately the activity of the Laboratory of Experimental Astrophysics has been supported by the Italian Ministero dell'Istruzione, dell'Università e della Ricerca (MIUR).

\section{References}

Allamandola, L.J., Sandford, S.A., Valero, G.J. 1988, Icarus 76, 225

Allamandola, L.J., Sandford, S.A., Tielens, A.G.G.M., Herbst, T.M. 1992, ApJ 399, 134

Baratta, G.A. \& Palumbo, M.E. 1998, J. Optical Soc. Am. A 15, 3076

Baratta, G.A., Leto, G., Spinella, F., Strazzulla, G., Foti, G. 1991, A $\& A$ 252, 421

Baratta, G.A., Castorina, A.C., Leto, G., Palumbo, M.E., Spinella, F., Strazzulla, G. 1994, Planet. Space Sci. 42, 759

Baratta, G.A., Palumbo, M.E., Strazzulla, G. 2000, A\&SA 357, 1045

Baratta, G.A., Leto, G., Palumbo, M.E. 2002, A\&A 384, 349

Bar-Nun, A., Herman, G., Laufer D., Rappaport, M.L. 1985, Icarus 63, 317

Bergin, E., Melnick, G.J., Gerakines, P.A., Neufeld, D.A., Whittet D.C.B. 2005, ApJ in press

Boogert, A.C.A., Schutte, W.A., Helmich, F.P., Tielens, A.G.G.M., Wooden, D.H. 1997, A\&\&A 317,929

Brown, W.L., Lanzerotti, L.J., Johnson, R.E. 1982, Science 218, 525

Brucato, J.R., Castorina, A.C., Palumbo, M.E., Satorre, M.A., Strazzulla G. 1997, Planet. Space Sci. 45,835

Chiar, J.E., Adamson, A.J., Kerr, T.H., Whittet, D.C.B. 1994, ApJ 426, 240

Collings, M.P., Anderson, M.A., Chen, R. Dever, J.W., Viti, S., Williams, D.A., McCoustra M.R.S. 2004, Mon. Not. R. Astron. Soc. 354, 1133

Cottin, H., Szopa, C., Moore, M.H. 2001, ApJ 561, L139

Cottin, H., Moore, M.H. Bénilan, Y. 2003, ApJ 590, 874

Dartois, E., Schutte, W., Geballe T.R., Demyk, K., Ehrenfreund P., d'Hendecourt L. 1999a, $A \mathscr{G} A$ (Letters) 342, L32

Dartois, E., Demyk, K., d'Hendecourt, L., Ehrenfreund, P. 1999b, A\&\&A 351, 1066

Dartois, E. \& d'Hendecourt, L. 2001, A\& A 365, 144

Ehrenfreund, P., Dartois, E., Demyk, K., d'Hendecourt, L. 1998, A\&A (Letters) 339, L17

Ferini, G., Baratta, G.A., Palumbo, M.E. 2004, A\&A 414, 757

Gerakines, P.A. \& Moore, M. H. 2001, Icarus 154, 372

Gerakines, P.A., Schutte, W.A., Ehrenfreund P. 1996, A\&A 312, 289

Gerakines, P.A., Whittet, D.C.B., Ehrenfreund, P., Boogert, A.C., Tielens, A.G.G.M., Schutte, W.A., Chiar, J.E., Van Dishoeck, E.F., Prusti, T., Helmich, F.P., de Graauw, Th. 1999, ApJ 522, 357

Gerakines, P.A., Moore, M.H., Hudson, R.L. 2000, A\& $A$ 357, 793

Gerakines, P.A., Moore, M.H., Hudson, R.L. 2004, Icarus 170, 202

Gibb, E.L., Whittet, D.C.B., Boogert A.C.A., Tielens, A.G.G.M. 2004, ApJ (Supplement Series) 151,35

Greenberg, M. 1982, in: L.L. Wilkening (ed.), Comets, (The University of Arizona Press, Tucson) p.131

Hagen, W., Allamandola, L.J., Greenberg, J.M. 1979, Ap\&SS, 65, 215

Hagen, W., Tielens, A.G.G.M., Greenberg, J.M. 1981, Chem. Phys. 56, 267

Horn, A., Mollendal, H., Sekiguchi, O., Uggerud, E., Roberts, H., Herbst, E., Viggiano, A.A., Fridgen, T.D. 2004, ApJ, 611, 605

Hudson, R.L. \& Moore, M.H. 2000, Icarus 145, 661

Hudson, R.L. \& Moore, M.H. 2002, ApJ 568, 1095

Ikeda, M., Ohishi, M., Nummelin, A., Dickens, J.E., Bergman, P., Hjalmarson, A., Irvine, W.M. 2001, ApJ, 560, 792

Leto, G. \& Baratta, G.A. 2003, A\&A 397, 7

Loeffler, M.J., Baratta, G.A., Palumbo, M.E., Strazzulla, G., Baragiola, R. 2005, A\&SA 435, 587

Mathis, J.S., Mezger, P.G., Panagia, N. 1983, A\&\&A 128, 212 
Mennella, V., Baratta, G.A., Esposito, A., Ferini, G., Pendleton, Y.J. 2003, ApJ 587, 727

Mennella, V., Palumbo, M.E., Baratta, G.A. 2004, ApJ 615, 1073

Moore, M.H. 1999, in: L. d'Hendecourt, C. Joblin, \& A. Jones (eds.), Solid Interstellar Matter: the ISO Revolution, (Springer-Verlag, New York), p. 199

Moore, M.H. \& Hudson, R.L. 1992, ApJ 401, 353

Moore, M.H., Donn, B., Khanna, R., A'Hearn M. F. 1983, Icarus 54, 388

Moore, M.H., Hudson, R.L. Gerakines, P.A. 2001, Spectrochim. Acta A 57, 843

Nummelin, A., Dickens, J.E., Bergman, P., Hjalmarson, A., Irvine, W.M., Ikeda, M., Ohishi, M. 1998, A\&A 337, 275

Palumbo, M.E., Baratta G.A. 2000, A\&A 361, 298

Palumbo, M.E., Castorina, A.C., Strazzulla, G. 1999, A\&A 342, 551

Palumbo, M.E., Ferini, G., Baratta G.A. 2004, Adv. Space Res. 33, 49

Prasad, S.S., \& Tarafdar, S.P. 1983, ApJ 267, 603

Roser, J. E., Vidali, G., Manicò, G., \& Pirronello, V. 2001, ApJ, 555, L61 vol. III, ed. K. N. Rao (Academic Press, London), 111

Strazzulla, G. \& Johnson, R.E. 1991, in: R. Newburg jr., M. Neugebaur, \& J. Rahe (eds.), Comets in the Post-Halley Era, (Kluwer, Dordrecht) p.243

Strazzulla, G., Calcagno, L., Foti, G. 1983, Mon. Not. R. Astron. Soc. 204, 59p

Strazzulla, G., Brucato, J.R., Palumbo, M.E., Satorre, M.A. 1997, A\&A 321, 618

Strazzulla, G., Baratta, G.A., Palumbo, M.E. 2001, Spectrochim. Acta A 57, 825

Tielens, A.G.G.M. \& Allamandola, L. J. 1987, in: G. E. Morfill, \& M. Scholer (eds.), Physical processes in interstellar clouds, (Reidel, Dordrecht) p.333

Watanabe, N. \& Kouchi, A. 2002, ApJ 567, 651

Westley, M. S., Baragiola, R. A., Johnson, R. E., \& Baratta, G. A. 1995a, Nature, 373, 405

Westley, M. S., Baragiola, R. A., Johnson, R. E., \& Baratta, G. A. 1995b, Planet. Space Sci., 43, 1311

Ziegler, J.F., J.P. Biersack, U. Littmark, (eds.), 1985, The Stopping and Range of Ions in Solids, Pergamon Press, New York 